\title{
Immunotherapeutic efficacy of a Lactobacillus casei lysate as an adjuvant combined with a heated-4T1 mammary carcinoma cell lysate in a murine model of breast cancer
}

\author{
Ruhollah Dorostkar ${ }^{\mathrm{a}}$, Mohammad Sadegh Hashemzadeha, Sajjad Jafarib, Mahdi Tat ${ }^{\mathrm{a}}$, Majdedin Ghalavand, \\ Mohammad Hossein Asghari ${ }^{\mathrm{c}, \mathrm{d}}$, Milad Moloudizargari ${ }^{\mathrm{e}}$ \\ a Applied Virology Research Center, Baqiyatallah University of Medical Sciences, Tehran 14351, Iran \\ ${ }^{b}$ Faculty of Veterinary Medicine, Urmia University, Urmia 57153, Iran \\ 'Department of Pharmacology, Faculty of Medicine, Babol University of Medical Sciences, Babol \\ 47176, Iran \\ ${ }^{d}$ Department of Toxicology and Pharmacology, Faculty of Pharmacy and Pharmaceutical Sciences \\ Research Center, Tehran University of Medical Sciences, Tehran 14155, Iran \\ eStudent Research Committee, Department of Immunology, School of Medicine, Shahid Beheshti \\ University of Medical Sciences, Tehran 19839, Iran
}

\begin{abstract}
Background: Immunotherapy, during which the immune system of the patient is manipulated to act against tumors has been among the most successful methods in the treatment of breast cancer, a leading cause of mortality among women worldwide.

Objectives: To investigate the immunotherapeutic efficacy of Lactobacillus casei lysate as an adjuvant in combination with a heated-4T1 mammary carcinoma cell lysate in a model of breast cancer.

Methods: After ethics committee approval of all animal procedures, a murine model of breast cancer was induced in BALB/c mice using 4T1 cells. These mice were immunized with a combination of lysates of heated 4T1 cells and L. casei. Subsequent changes in tumor size and weight, and the production of TNF- $\alpha$, IL-2, IL-12, IL-17, and IL13 were measured. Lung weights were measured as an indicator of metastasis to other organs.

Results: The tumor size and weight in mice immunized with the combined vaccine were significantly reduced compared with controls. The combined immunotherapy altered the pattern of cytokine production to the advantage of antitumor immunity, and was significantly more potent than immunization with heated-4T1-cell lysate or L. casei lysate alone.

Conclusions: Coadministration of $L$. casei lysate enhanced the immunotherapeutic efficacy of the heated-4T1cell lysate as a source of tumor-associated antigens. L. casei can potentially be used as an adjuvant combined with sources of tumor antigens in the treatment of cancers, and as a safe alternative to the current adjuvants that cause greater irritation to hosts. Further studies are required to clarify the mechanisms underlying these effects.
\end{abstract}

Keywords: Cancer immunotherapy, HSP, Lactobacillus casei, TNF- $\alpha$, tumor, vaccine

Malignancies are among the major health problems for humans and not only cause widespread mortality, but also impose a heavy financial burden on health care systems of all societies [1]. Breast cancer

Correspondence to: Milad Moloudizargari, Department of Immunology, School of Medicine, Shahid Beheshti University of Medical Sciences, Tehran 198396, Iran. E-mail: miladmoludi@ sbmu.ac.ir is one of the most common malignancies, accounting for approximately one-third of all cancers occurring in women. It is expected that 1 in every 8 women in the USA will develop breast cancer in her lifetime [2]. Increasing prevalence of breast cancer and its immense impact on the quality of life of affected individuals [3] has led to numerous efforts in its treatment, control, and prevention. 
A relatively new understanding of the interactions between the immune system and tumors, has given rise to the development of a modern therapeutic approach to malignancies known as immunotherapy [4]. Cancer immunotherapy includes a range of manipulations during which a potent antitumor immune response is activated [5]. This can target both the innate and adaptive immunities, and can be achieved either by augmenting the antitumor response or the disrupting the regulatory mechanisms used by the immune system [6]. This can be elicited to various extents by distinct methods such as the delivery of adjuvants to the tumor milieu and immunization with tumor associated antigens (TAA) in isolated form or as whole cell preparations. For this purpose, various types of vaccines contain tumor cell lysates [7] and heated tumor cell lines have been used as rich sources of TAAs. For instance, dendritic cell-based immunotherapy of tumors has been shown to be a promising method to provoke the antitumor immune response, especially when incorporated with whole tumor antigens [8]. Because of a variety of identified and unidentified epitopes that could be recognized by $\mathrm{CD}^{+}$and $\mathrm{CD} 8^{+} \mathrm{T}$ cells, whole tumor antigens seem to be more effective compared with individual antigens, such as tumor-derived peptides or recombinant tumor proteins [9].

The 4T1 mammary carcinoma cell line is widely used in animal model breast cancer studies because of its strong tumorigenic and highly metastatic properties, and can be readily transplanted into animals where it can develop palpable tumors $[3,5,10,11]$.

According to a definition by the World Health Organization, probiotics are "live microorganisms that, when administered in adequate amounts, confer a health benefit on the host" [12]. Lactobacilli are one of the most frequently used probiotics for controlling intestinal microflora with the potential to suppress harmful bacteria with high safety [12]. Among several strains, Lactobacillus casei possess immunomodulatory properties $[13,14]$. Recent studies have focused on the potential of $L$. casei as an adjuvant in cancer therapy $[15,16]$.

The present study aimed to investigate the immunotherapeutic efficiency of a new cancer vaccine using a combination of sublethally-heated-4T1-cell lysate as a source of TAAs and L. casei lysate as an adjuvant by comparing with the individual administration of each component in 4T1-induced mice models of breast cancer.

\section{Materials and methods \\ Reagents}

Phytohemagglutinin (PHA), dioxan, and phosphate-buffered saline (PBS) were purchased from Sigma-Aldrich (St. Louis, MO, USA). Fetal calf serum (FCS) and Dulbecco's modified Eagle's medium (DMEM) were purchased from Gibco/Life Technologies (Gaithersburg, MD, USA), and the enzyme-linked immunoassay (ELISA) kits were from Qiagen (Hilden, Germany).

\section{Tumor cell culture}

The 4T1 cell line was provided by the Pasteur Institute of Iran and cultures were grown in a humidified atmosphere at $37^{\circ} \mathrm{C}$ under an atmosphere containing $5 \% \mathrm{CO}_{2}$. The cells were maintained as monolayers in DMEM supplemented with $10 \%$ FBS. To prepare the vaccine, the cells were treated as follows. To induce the production of heat shock proteins (HSPs) in the tumor cells, the cells were treated with sublethal heat at $43^{\circ} \mathrm{C}$ for $30 \mathrm{~min}$ in a bain-marie, as described previously by Huang et al. [17]. The cells were then incubated for $12-48 \mathrm{~h}$ and a suspension of $1 \times 10^{6} \mathrm{cells} / \mathrm{mL}$ was prepared [18]. Subsequently, the cells underwent 4 freeze-thaw cycles by placing them in liquid nitrogen and alternately incubating at $37^{\circ} \mathrm{C}$ to produce the required cell lysate. As the final step, the lysate was centrifuged at $4000 \mathrm{rpm}$ and the supernatant was passed through a $0.2 \mu \mathrm{m}$ filter to be used as the vaccine [19].

\section{L. casei culture and lysate preparation}

L. casei (American Type Culture Collection No. 393) were purchased from the Pasteur Institute of Iran and were cultivated in deMan-Rogosa-Sharpe medium (MRS) at $37^{\circ} \mathrm{C}$ for $24 \mathrm{~h}$ under anaerobic conditions. After the growth of bacteria, which was determined from the opacity of the culture, the culture was centrifuged at $3000 \mathrm{rpm}$ for 20 minutes at $4^{\circ} \mathrm{C}$. The cell pellet was washed twice with phosphatebuffered saline (PBS), resuspended in $1 \mathrm{~mL}$ of the same buffer and stored at $-80^{\circ} \mathrm{C}$ overnight. To obtain the cellular extract, the method described by Lebendiker was followed [20]. Briefly, after thawing the bacteria, $100 \mu \mathrm{L}$ of lysing buffer was added to 1 $\mathrm{mL}$ of the bacterial suspension. The bacteria were lysed using a Soniprep 150 sonicator (MSE, London, UK). The obtained lysate was passed through a 0.2 $\mu \mathrm{m}$ filter and was used as the adjuvant. 


\section{Animal studies and tumor induction}

Forty BALB/c mice, 6-8 weeks of age, were purchased from the Pasteur Institute of Iran and were acclimatized to their housing for one week. The animals were kept under standard housing conditions at a temperature of $22-24^{\circ} \mathrm{C}$ under a $12 \mathrm{~h}$ light-dark cycle and were allowed water and standard laboratory rodent food ad libitum during both the adaptation and experimental periods. All animal studies were performed in compliance with the regulations of the Ministry of Health, Iran and were approved by the Medical Ethics Committee of Urmia University for Animal Studies (approval No. 1432, October 16, 2015).

Subcutaneous challenge of the animals in all groups with $50 \mu \mathrm{L}$ PBS suspension containing $1 \times 10^{4}$ viable tumor cells was considered as the onset of the experiment. The animals were observed for the development of palpable tumors and were equally distributed in 4 groups of 10 following confirmation of tumor development, including a control group, and 3 treatment groups each receiving immunization with a different vaccine.

\section{Vaccination}

Vaccination was started when all mice had developed a palpable tumor (day 12). The left flank of each mouse was chosen as the injection site and all vaccines were delivered subcutaneously. Each mouse in the control group was immunized with 2 injections $100 \mathrm{~L}$ of PBS at a one-week interval. Mice in the first treatment group received heated-4T1-cell lysate alone; the mice were immunized with 2 injections of the freeze-thaw lysate of $10^{4}$ heated 4T1 cells in $100 \mu \mathrm{L}$ PBS at a one-week interval. Mice in second treatment group were immunized with 2 injections of $L$. casei lysate in $100 \mu \mathrm{L}$ PBS alone at a one-week interval. Mice in the third group were immunized with a combination of heated-4T1-cell lysate and L. casei lysate in $100 \mu \mathrm{L}$ PBS at a oneweek interval.

\section{Evaluation of tumor growth and metastasis}

At the end of the experimental period, 62 days from the onset challenge by viable tumor cells, the mice were humanely killed and following the aseptic isolation of their spleens for cell culture, the tumors were measured for size and weighed as 2 indicators of tumor growth. The size was measured using the formula of an ellipsoid (length $x$ width $\times$ height $\times$
0.5236 ) and then expressed as $\mathrm{mm}^{3}$. Each tumor was resected and weighed separately and the weights were recorded in grams.

\section{Cytokine assays}

In brief, splenocytes were aseptically isolated from mice and single-cell suspensions of splenocytes were prepared in DMEM supplemented with 10\% FCS. The red blood cells were removed using a lysis buffer $[21,22]$. Next, cell suspensions $\left(2 \times 10^{6}\right.$ cells $\left./ \mathrm{mL}\right)$ were incubated in 24-well culture plates and pulsed with antigens derived from tumor cells by the freezethaw method $(100 \mathrm{~g} / \mathrm{mL})$ and $25 \mu \mathrm{L}$ PHA solution $(1 \mathrm{mg} / \mathrm{mL})$. Tumor antigen was prepared as described previously. The culture supernatants were collected after $72 \mathrm{~h}$. Tumor necrosis factor (TNF)- $\alpha$, interleukin (IL)-2, IL-12, IL-17, and IL-13 levels were determined by ELISA according to the assay manufacturer's instructions.

\section{Statistical analysis}

Statistical analyses were performed using a one-way analysis of variance (ANOVA) following by a Tukey test to determine differences between the different groups. Results are shown as mean \pm standard deviation (SD). $P<0.05$ was considered significant.

\section{Results}

Tumor size and weight studies revealed that immunization of the animals with a combination of L. casei and 4T1 cell lysates can significantly decrease tumor growth, compared with the control group (Figure 1). Although treatment with heated-4T1-cell lysate alone also decreased the tumor size compared with the control group, the significance of the difference between the heated-4T1-cell lysate and combinedtreatment groups indicated that treatment with heated4T1-cell lysate alone is not as effective as the combined treatment in controlling the growth of the tumors. Treatment with heated $L$. casei alone did not produce any significant changes in the tumor size. Like the combined immunization, immunizations with $L$. case $i$ and heated-4T1-cell lysates alone also decreased the tumor weights. However, immunization of the mice with a combination of $L$. case $i$ and heated-4T1-cell lysates had the most potent effect on tumor weight and size. 

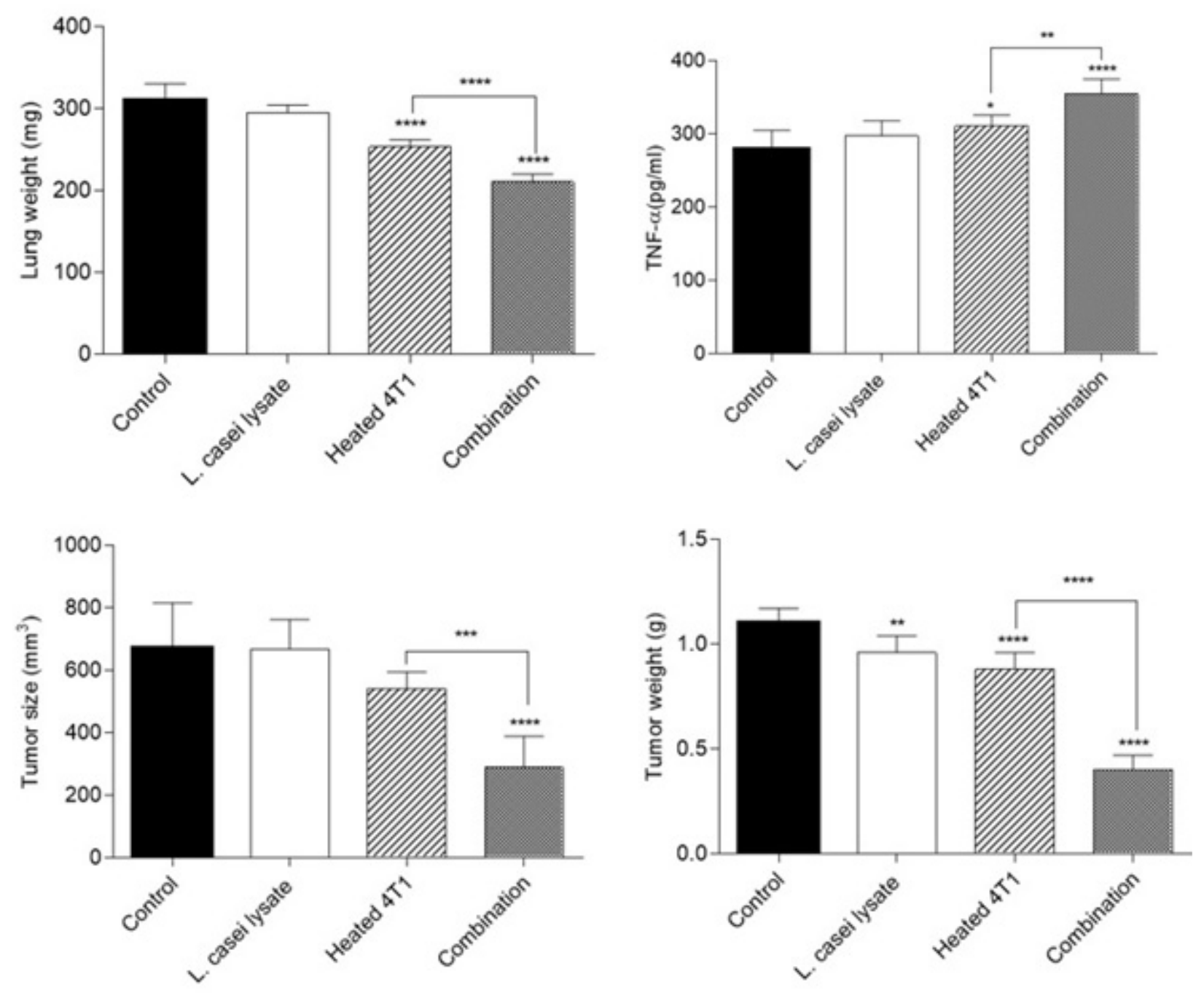

Figure 1. Comparison of lung weight (upper left), tumor necrosis factor (TNF)- $\alpha$ levels (upper right), tumor size (lower left), and tumor weight (lower right), and after immunization with a heated-4T1-cell lysate alone (hatched bar), the L. casei lysate alone (open bar), and their combination (crosshatched bar). ${ }^{*} P<0.05,{ }^{* *} P<0.01,{ }^{* * *} P<0.001$, and ${ }^{* * * *} P<0.0001$.

Studies of metastasis showed that treatment with the combination of heated-4T1-cell lysate and L. casei lysate, and treatment with heated-4T1-cell lysate alone could hinder metastasis to the lung as determined by the significantly lower lung weight compared with controls $(P<0.0001)$. The combined immunotherapy showed a more potent effect than immunotherapy with the heated-4T1-cell lysate alone.

The results of all ex vivo cytokine assays included in this study were in parallel with the results for tumor growth and metastasis, indicating that the combined vaccine had the strongest antitumor properties. The splenocytes of the mice from the combined treatment group significantly upregulated production of TNF- $\alpha$, IL-2, IL-12, and IL-17, while they significantly downregulated production of IL-13, compared with splenocytes from mice in the control group. Immunization with the heated-4T1-cell lysate alone resulted in significant upregulation of IL-2, IL-17, and TNF- $\alpha$, while it downregulated the production of IL13. Its effect on IL-2 was comparable to that of the combination vaccine. With the exception of IL-2, immunization with the $L$. casei lysate alone did not produce any significant changes in the levels of any of the cytokines measured (Figure 2). 

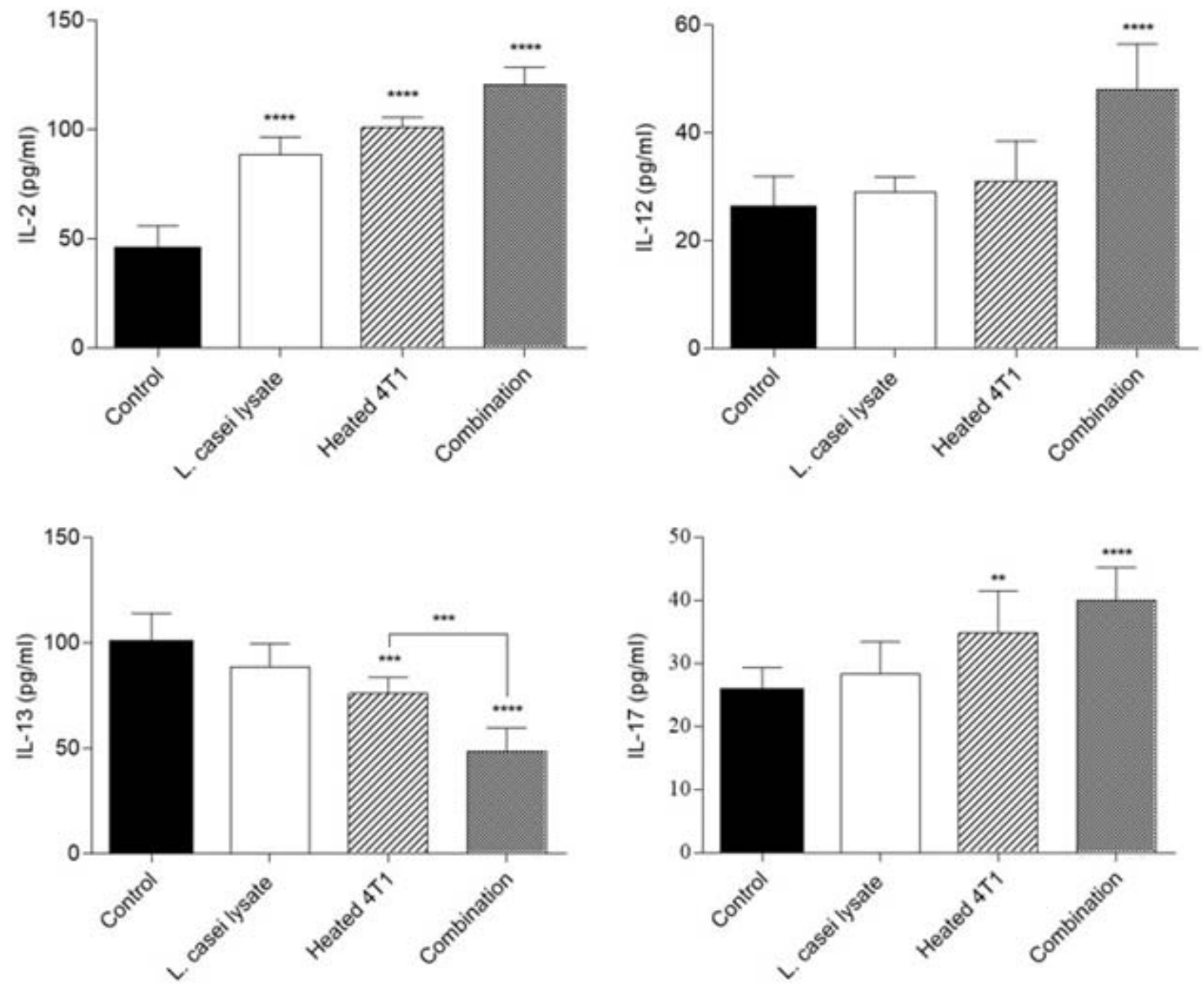

Figure 2. Levels of IL-2 (upper left), IL-12 (upper right), IL-13 (lower left), and IL-17 (lower right) after immunization with a heated-4T1-cell lysate alone (hatched bar), the $L$. casei lysate alone (open bar), and their combination (crosshatched bar). ${ }^{*} \mathrm{P}<0.05,{ }^{* *} \mathrm{P}<0.01,{ }^{* * *} \mathrm{P}<0.001$, and ${ }^{* * * *} \mathrm{P}<0.0001$.

\section{Discussion}

It has been previously shown that exposure of 4T1 cells to sublethal heat not only impairs their ability to proliferate and metastasize, but also results in increased expression of HSP72, a member of the HSP70 family [23]. Moderate hyperthermia $\left(39^{\circ} \mathrm{C}\right.$ to $43^{\circ} \mathrm{C}$ ) induces a $500-1000$-fold increase in the in vitro expression of a heterologous gene in the promoter of HSP70 [17]. This increase might be responsible, at least in part, for the increased HSP production following exposure to sublethal heat. The HSP70 family facilitates the recognition and uptake of TAAs by antigen presenting cells, thus enhancing the immunogenicity of tumors [24]. Therefore, the 4T1 cell line used in this study was subjected to sublethal heat to achieve the desired stimulus of the immune response by the tumor cell lysate, and control of the growth of the tumors. Changes in tumor weight and size were deemed as indices of tumor growth. As shown by Figure 1, combined immunization with heated-4T1-cell and $L$. casei lysates was effective in controlling tumor growth. Although tumor weights were similarly reduced after immunization of the mice with the heated-4T1-cell lysate alone and $L$. casei lysate alone; the strongest effects were observed after immunization with the combined vaccine.

Stimulus of the innate immune system was not found, no specific immune response is seen initiated by such treatment [25]. Pattern recognition receptors such as toll-like receptors are functional compartments of the immune system that recognize specific microbial patterns and in response to 
this recognition, induce the expression of certain costimulatory molecules and cytokines that are involved in initiating potent antitumor $\mathrm{Th}_{1}$ and cytotoxic $\mathrm{T}$ cell responses [26]. Regrettably, tumor cells lack the ability to induce the expression of such costimulatory molecules, which necessitates the application of adjuvants with an antigen source to elicit potent antitumor responses. Adjuvants modulate the immune reaction to specific antigens [27]. A desirable adjuvant in cancer immunotherapy is one that can polarize the immune response towards $\mathrm{Th}_{1}$-mediated immunity [28]. Another concern regarding the application of adjuvants is the level of safety for use in humans [27]. Although various compounds such as Th ${ }_{1}$-promoting cytokines [29] and CpG DNA [30] have been shown to possess such adjuvant-like properties; finding adjuvants with low toxicity remains a challenge for researchers.

L. casei are a safe probiotic, and possess antitumor effects [15]. In the present study, we evaluated lysates of $L$. casei for possible adjuvantlike effects against 4T1-induced mammary tumors. L. casei lysate in combination with the heated-4T1cell lysate significantly upregulated the production

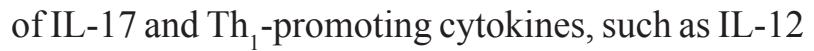
and IL-2 (Figure 2). The combination also upregulated the levels of TNF- $\alpha$, which plays a crucial role in antitumor immunity (Figure 1). By contrast, IL-13 production was downregulated following immunization with the combined vaccine, enhancing the antitumor immune response. Similar, but less potent effects were found for immunization with the heated-4T1-cell lysate alone. However, with the exception of IL-2, no significant changes in cytokine levels were found after immunization with the $L$. casei lysate alone.

L. casei can induce apoptosis in a colorectal cancer cells in vitro [3]. In vivo the antitumor effects of $L$. casei on colorectal cancer are mediated by modulation of regulatory T-cells towards a $\mathrm{Th}_{17}$-biased immune response [31]. These findings are consistent with the increased secretion of IL-17 seen following immunization with the combined vaccine in the present study.

The antitumor efficacy of this strain of Lactobacilli has also been shown against gastric cancer with a similar mechanism proposed to induce apoptosis. The efficacy was attributed to inhibiting nuclear factor (NF)- $\mathrm{KB}$ and mTOR-mediated signaling (15). The direct treatment of a cervical cancer cell line with a cell-free extract of $L$. casei did not have any significant inhibitory effect on the proliferation of the cells [16]. This suggests that the effects observed with the L. casei lysate in the present study are because of an adjuvant-like activity in combination with the heated4T1-cell lysate rather than its direct effects on tumor cells.

In a rat model of mammary cancer induced by 2-amino-1-methyl-6-phenylimidazo[4,5-b]pyridine, the coadministration of $L$. casei Shirota with soymilk was found to suppress tumor growth, and thus enhance the prophylactic effect of soymilk against the tumors by reducing the number of estrogen receptors and Ki-67 positive cells within the tumor [32]. Oral administration of milk fermented with $L$. casei inhibited the growth and metastasis of breast cancer cells in mice, characterized by a decrease in tumor vascularity, decreased shedding of tumor cells, and decreased metastasis to the lung [33]. The partial cytokine profile determined in the present study showed the antitumor effect of $L$. casei lysate combined with heated-4T1-cell lysate on the immune response, which is consistent with the results of the previous studies. The lung weights as an indicator of the metastasis of the induced tumors was consistent with these findings indicating that immunization of the mice with the combined vaccine significantly potentiated the inhibitory effect of heated-4T1-cell lysate on tumor metastasis. One of the inevitable complications of the use of self-antigens in combination with adjuvants to combat tumors is the possibility of autoimmune responses that might be evoked in the host receiving the immunotherapy. Although some layers of selectivity can be achieved to discriminate between self and tumor antigens during the antigen processing and antigen presentation by antigen presenting cells [34], targeting of self-antigens in tumor immunotherapy remains a challenge, especially when a wide array of tumor antigens is delivered in an immunological context. Nevertheless, there have been numerous efforts to specifically direct the stimulated antitumor response towards the tumor cells. Isolation of specific antigens, which are expressed differently on healthy and tumor cells [35], and the application of enhanced delivery protocols using nanoparticles as antigen carriers [36] are among these efforts. The amount of antigen that is introduced to the host is also a main determinant of the quality and intensity of any immune response [37]. The route of vaccination, and the duration and frequency of antigen exposure are among other important factors affecting 
such a response [38]. Thus, dose adjustment of the vaccine and a proper administration protocol also may influence the selectivity of the elicited response, and optimal regimens remain to be elucidated in future studies.

\section{Conclusion}

The present study shows that $L$. casei lysates can be potentially used to boost antitumor immune responses. This effect of $L$. casei lysate is adjuvantlike rather than a direct antitumor activity, making it a desirable candidate for enhancing the efficacy of compounds used in cancer therapy when used in combination with sources of TAAs. Further research is required to confirm the safety and quality of $L$. casei and its derivatives as novel adjuvants in cancer immunotherapy.

\section{Conflict of interest statement}

The authors declare that there is no conflict of interest in this research.

\section{References}

1. Siegel RL, Miller KD, Jemal A. Cancer statistics, 2015. CA Cancer J Clin. 2015; 65:5-29.

2. DeSantis C, Ma J, Bryan L, Jemal A. Breast cancer statistics, 2013. CA Cancer J Clin. 2014; 64:52-62.

3. Howard-Anderson J, Ganz PA, Bower JE, Stanton AL. Quality of life, fertility concerns, and behavioral health outcomes in younger breast cancer survivors: a systematic review. J Natl Cancer Inst. 2012; 104: 386-405.

4. Finn OJ. Cancer immunology. New Engl J Med. 2008; 358:2704-15.

5. Dougan M, Dranoff G. Immunotherapy of cancer. In: Wang R-F, editor. Innate immune regulation and cancer immunotherapy. New York: Springer; 2012. p. 391-414.

6. Dougan M, Dranoff G. Immune therapy for cancer. Annu Rev Immunol. 2009; 27:83-117.

7. Mueller M, Reichardt W, Koerner J, Groettrup M. Coencapsulation of tumor lysate and $\mathrm{CpG}-\mathrm{ODN}$ in PLGA-microspheres enables successful immunotherapy of prostate carcinoma in TRAMP mice. J Controlled Release. 2012; 162:159-66.

8. González FE, Gleisner A, Falcón-Beas F, Osorio F, López MN, Salazar-Onfray F. Tumor cell lysates as immunogenic sources for cancer vaccine design. Hum Vaccin Immunother. 2014; 10:3261-9.

9. Chiang CL-L, Coukos G, Kandalaft LE. Whole tumor antigen vaccines: where are we? Vaccines. 2015; 3:
$344-72$.

10. Guo QT, Li X, Yang Y, Wei J, Zhao Q, Luo FR, et al. Enhanced 4T1 breast carcinoma anticancer activity by co-delivery of doxorubicin and curcumin with core-shell drug-carrier based on heparin modified poly (L-lactide) grafted polyethylenimine cationic nanoparticles. J Biomed Nanotechnol. 2014; 10:227-37.

11. Motlagh BM, Ahangaran NA, Froushani SMA. Calcitriol modulates the effects of bone marrow-derived mesenchymal stem cells on macrophage functions. Iran J Basic Med Sci. 2015; 18:672-76.

12. Gill H, Prasad J. Probiotics, immunomodulation, and health benefits. In: Bösze Z, editor. Bioactive components of milk. Adv Exp Med Biol vol. 606 . New York: Springer; 2008. p. 423-54.

13. Dong H, Rowland I, Thomas LV, Yaqoob P. Immunomodulatory effects of a probiotic drink containing Lactobacillus casei Shirota in healthy older volunteers. Eur J Clin Nutr. 2013; 52:1853-63.

14. Matsuzaki T. Immunomodulation by treatment with Lactobacillus casei strain Shirota. Int J Food Microbiol. 1998; 41:133-40.

15. Hwang JW, Baek Y-M, Yang KE, Yoo H-S, Cho C-K, Lee Y-W, et al. Lactobacillus casei extract induces apoptosis in gastric cancer by inhibiting NF- $\kappa \mathrm{B}$ and mTOR-mediated signaling. Integr Cancer Ther. 2013; 12:165-73.

16. Kim S-N, Lee WM, Park KS, Kim JB, Han DJ, Bae J. The effect of Lactobacillus casei extract on cervical cancer cell lines. Contemp Oncol (Pozn). 2015; 19: 306-12.

17. Huang Q, Hu JK, Lohr F, Zhang L, Braun R, Lanzen J, et al. Heat-induced gene expression as a novel targeted cancer gene therapy strategy. Cancer Res. 2000; 60:3435-9.

18. Steinman L, Zamvil SS. How to successfully apply animal studies in experimental allergic encephalomyelitis to research on multiple sclerosis. Ann Neurol. 2006; 60:12-21.

19. Delirezh N, Moazzeni SM, Shokri F, Shokrgozar MA, Atri M, Kokhaei P. Autologous dendritic cells loaded with apoptotic tumor cells induce $\mathrm{T}$ cell-mediated immune responses against breast cancer in vitro. Cell Immunol. 2009; 257:23-31.

20. Lebendiker M. The Wolfson Centre for Applied Structural Biology. Bacterial protein extraction - mini scale - Sonication. 2002. Available at http://wolfson. huji.ac.il/purification/TagProteinPurif/Lysis_ Bacterial_Cells.html. Accessed March 15, 2016.

21. Abtahi Froushani SM, Delirezh N, Hobbenaghi R, 
Mosayebi G. Synergistic effects of atorvastatin and all-trans retinoic acid in ameliorating animal model of multiple sclerosis. Immunol Invest. 2014; 43:54-68.

22. Jahangiri S, Abtahi Froushani SM, Delirezh N. Combination immunotherapy with extract of heated 4T1 and naloxone in mouse model of breast cancer. Turk J Med Sci. 2016; 46:518-23.

23. Bausero MA, Page DT, Osinaga E, Asea A. Surface expression of Hsp25 and Hsp72 differentially regulates tumor growth and metastasis. Tumor Biol. 2004; 25 : 243-51.

24. Vanbuskirk A, Crump BL, Margoliash E, Pierce S. A peptide binding protein having a role in antigen presentation is a member of the HSP70 heat shock family. J Exp Med. 1989; 170:1799-809.

25. Iwasaki A, Medzhitov R. Control of adaptive immunity by the innate immune system. Nat Immunol. 2015; 16: 343-53.

26. Tanimura N, Miyake K. Toll like receptors. In: Taniguchi $\mathrm{N}$ et al., editors. Glycoscience: biology and medicine. Tokyo: Springer; 2015. p. 707-12.

27. Mesa C, Fernández LE. Challenges facing adjuvants for cancer immunotherapy. Immunol Cell Biol. 2004; 82:644-50

28. Dredge K, Marriott JB, Todryk SM, Dalgleish AG. Adjuvants and the promotion of Th1-type cytokines in tumour immunotherapy. Cancer Immunol Immunother. 2002; 51:521-31.

29. Bobanga ID, Petrosiute A, Huang AY. Chemokines as cancer vaccine adjuvants. Vaccines. 2013; 1:444-62.

30. Bode C, Zhao G, Steinhagen F, Kinjo T, Klinman DM. CpG DNA as a vaccine adjuvant. Expert Rev Vaccines. 2011; 10:499-511.

31. Lenoir M, del Carmen S, Cortes-Perez NG, Lozano-
Ojalvo D, Muñoz-Provencio D, Chain F, et al. Lactobacillus casei BL23 regulates Treg and Th17 T-cell populations and reduces DMH-associated colorectal cancer. J Gastroenterol. 2016:1-12.

32. Kaga C, Takagi A, Kano M, Kado S, Kato I, Sakai M, et al. Lactobacillus casei Shirota enhances the preventive efficacy of soymilk in chemically induced breast cancer. Cancer Sci. 2013; 104:1508-14.

33. Aragón F, Carino S, Perdigón G, de Moreno de LeBlanc A. Inhibition of growth and metastasis of breast cancer in mice by milk fermented with Lactobacillus casei CRL 431. J Immunotherapy. 2015; 38:185-96.

34. Wagner SC, Riordan NH, Ichim TE, Szymanski J, Ma H, Perez JA, et al. Safety of targeting tumor endothelial cell antigens. J Trans Med. 2016; 14:1.

35. Coulie PG, Brichard V, Van Pel A, Wölfel T, Schneider J, Traversari C, et al. A new gene coding for a differentiation antigen recognized by autologous cytolytic T lymphocytes on HLA-A2 melanomas. J Exp Med. 1994; 180:35-42.

36. Babu A, Templeton AK, Munshi A, Ramesh R. Nanoparticle-based drug delivery for therapy of lung cancer: progress and challenges. J Nanomater. 2013; 2013:14

37. Pham VQ, Nguyen ST, Mai TT, Phan NK, Van Pham P. Breast cancer tumor growth is efficiently inhibited by dendritic cell transfusion in murine model. Biomed Res Ther. 2014; 1:85-92.

38. Levine B, Vaz N. Effect of combinations of inbred strain, antigen, and antigen dose on immune responsiveness and reagin production in the mouse. Int Arch Allergy Immunol. 1970; 39:156-71. 